\title{
Al Denoising to Accelerate Detector Simulation
}

\author{
Lena Franklin (University of Maryland), Kevin Pedro (Fermilab)
}

\section{Introduction}

Detector simulation is critical to experimental HEP; however this simulation (commonly done through toolkits such as Geant4) is computationally intensive. Performance can be improved somewhat through technical optimization, but more is needed. Using machine learning (ML) to accelerate simulation is a promising field, however efforts to use generative adversarial networks (GANs) or optimized autoencoders have faced issues. Using convolutional neural networks (CNNs) for denoising has been successful in non-HEP applications such as image processing. This poster investigates the efficacy of using CNNs to denoise Geant4 simulations. This could increase the accuracy of simulations performed under settings designed to increase computational efficiency.

\section{Network Architecture}

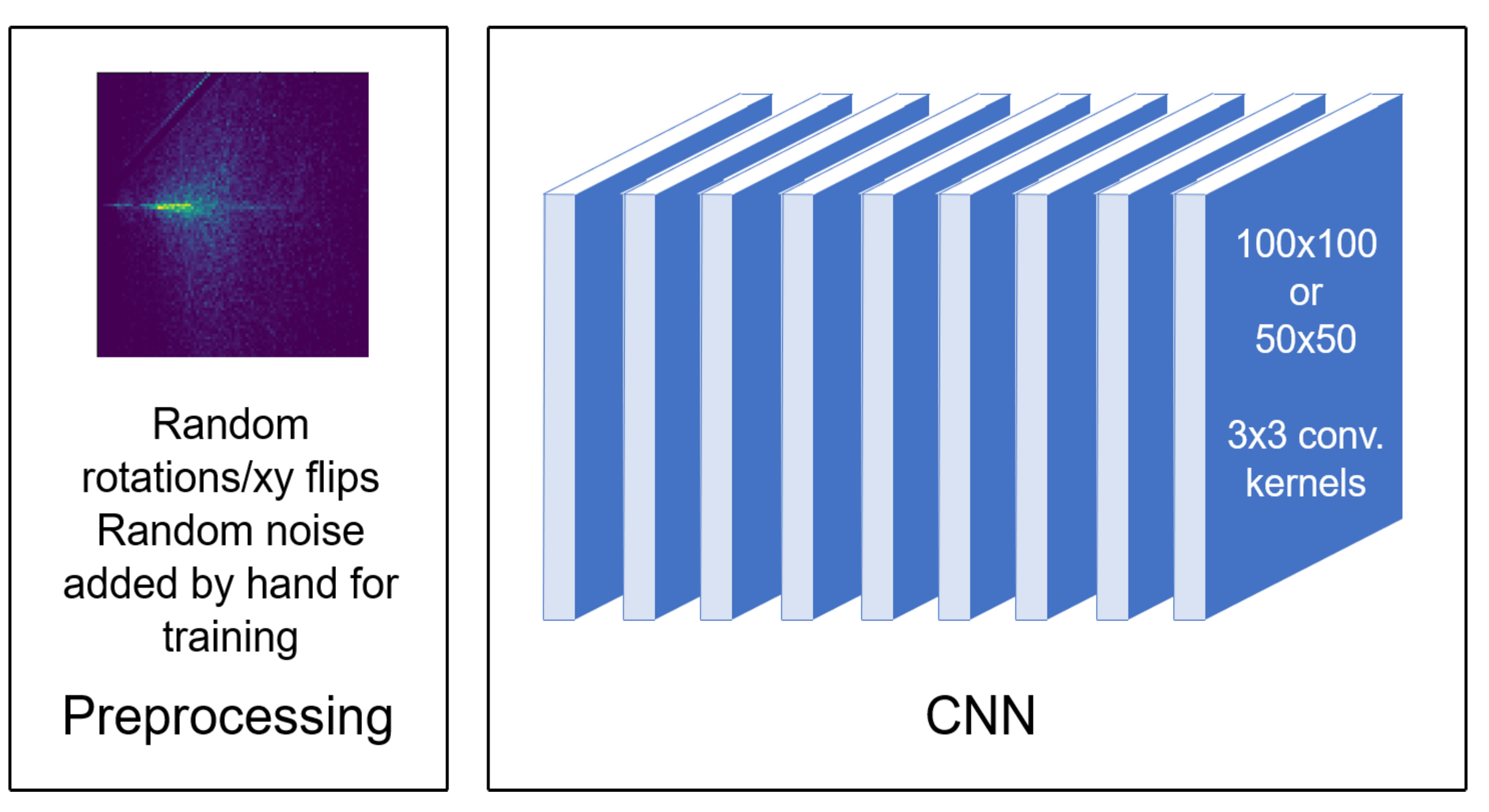

We use a network with nine convolutional layers and $3 \times 3$ pixe kernels and 100 feature vectors at each layer. A rectified linea unit (ReLU) activation function is applied at each layer. At each convolutional layer, appropriate padding is used to preserve the size of the input tensor in order to produce a denoised output tensor with the same dimensions as the original noisy tensor. The network accepts 2-dimensional input images with a single channel which records the energy deposited in an $x-y$ area of the simulated detector.

This work was supported in part by the U.S. Department of Energy, Office of Science, Office of Workforce Development for Teachers and Scientists (WDTS) under the Science Undergraduate Laboratory Internships Program (SULI).

\section{Loss and Training}

Our loss function prioritizes interesting regions of the simulation with high deposited energy by splitting the images into patches and returning the mean absolute error of the patch with the greatest loss, which corresponds to the patch with greater energy deposits.

Initial training was done with $100 \times 100$ pixel images generated from Geant4 simulations of single $100 \mathrm{GeV}$ photons in the CMS electromagnetic calorimeter. These images were randomly rotated during preprocessing

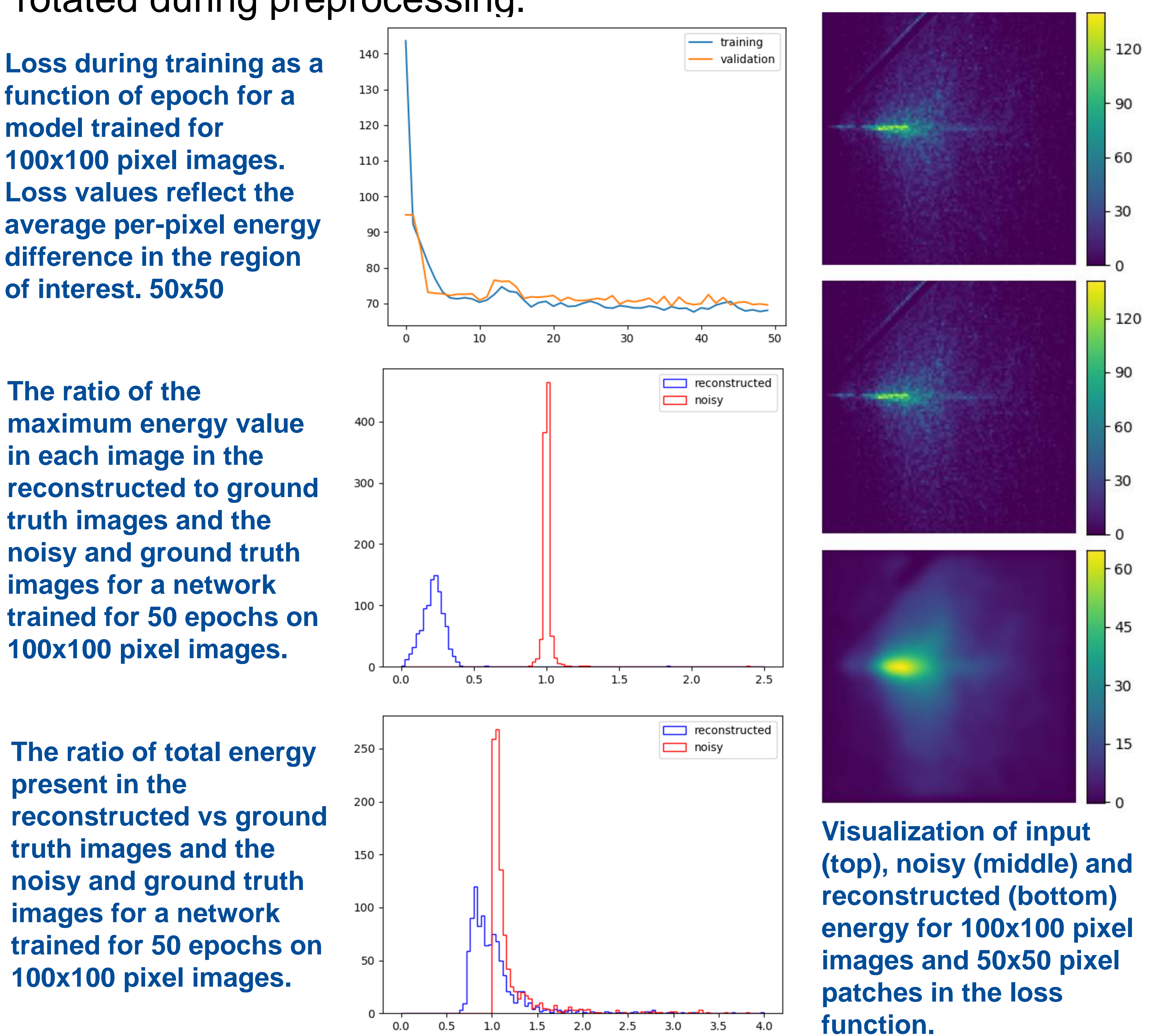

Optimizing Network Performance

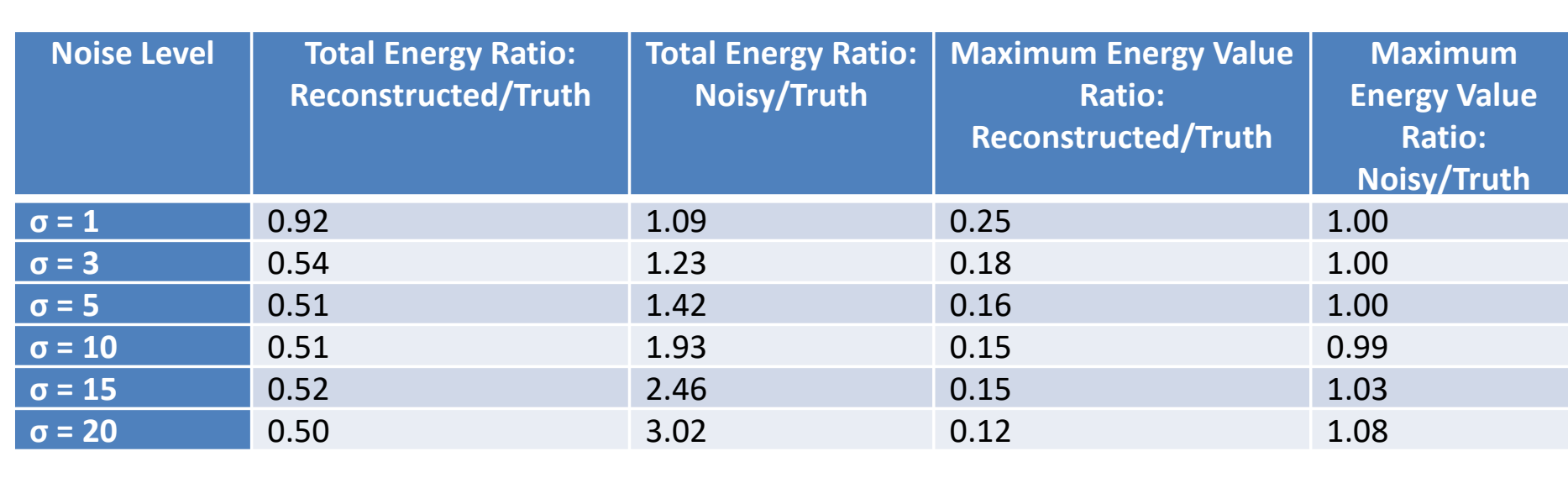
To improve the results achieved, we attempted to vary the parameters of the vary the parameters of the
network and of training, Results of a 50 epoch training of images of including the input noise leve of the training and validation

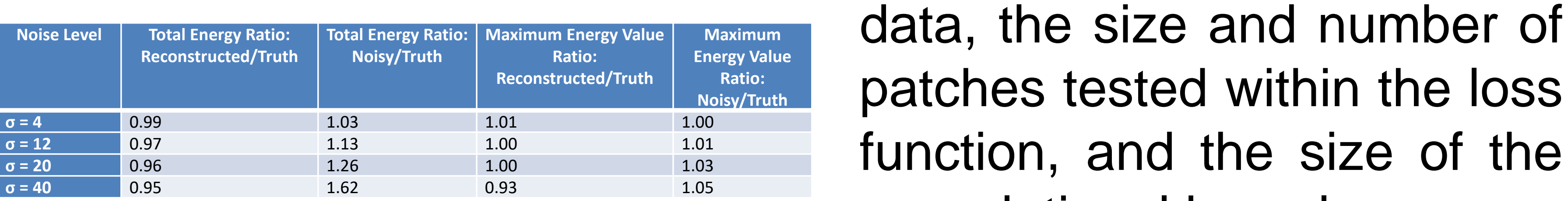
Results of a 50 epoch training of images of
$50 \times 50$ pixels each, training on full image.

\section{Optimizing Network Performance}

Performance was considerably improved when the size of the input was reduced from $100 \times 100$ to $50 \times 50$ pixels. We hypothesize this allowed the network to learn how to preserve desired features of the images, rather than those created by noise.

average per-pixel energy

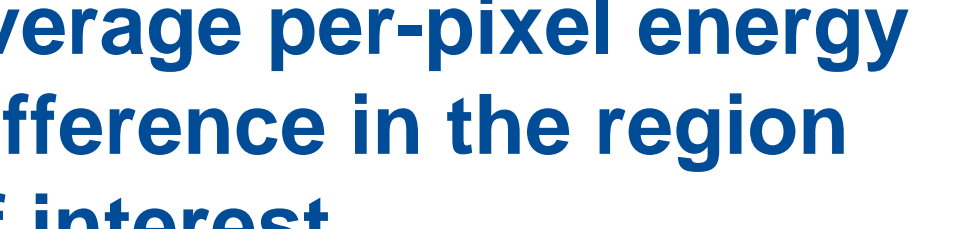

st.

The ratio of the maximum the reconstructed and reconstructed and sy and ground t rained for 50 epochs on $50 \times 50$ pixel images.

The ratio of total energy reconstrute truth images and the noisy und truth images for a network trained for 50

\section{Future Work}

- Try using a kernel-based prediction architectures in which the final network layer outputs a kernel of scalar weights to be applied to a noisy input area

- Use a 3-dimensional dataset which will more accurately rect the detector for simulation purposes

- Analyze modified loss functions \& preprocessing methods.

- Determine coarse settings to speed up Geant4 and generate noisy images instead of adding noise by hand.

- Add additional images channels to store additional information e.g. timing, PID, etc.

\section{References}

eve Bako, Thijs Vogels, et al. "Kernel-predicting convolutional networks for denoising
(1) (2017). 